\title{
A CHARACTERIZATION OF JANKO'S SIMPLE GROUP
}

\section{T. M. GAGEN ${ }^{1}$}

If $G$ is a finite group, we say that a series of subgroups $G=G_{0} \geqq G_{1}$ $\geqq \cdots \geqq G_{n}=1$ is a maximal series of length $n$ of $G$ if $G_{i}$ is a maximal subgroup of $G_{i-1}, 1 \leqq i \leqq n$. A subgroup $H$ of $G$ is called $m$ th maximal in $G$ if there exists at least one maximal series $H=G_{m} \leqq G_{m-1} \leqq \cdots$ $\leqq G_{0}=G$. Groups all of whose second, third and fourth maximal subgroups are invariant have been completely classified, see Janko [4], where the relevant results are enumerated. Further, those finite simple groups whose fifth maximal subgroups are trivial have been found by Janko [5].

Since Janko [6] has announced the discovery of a new simple group $J$, whose sixth maximal subgroups are all trivial, it may be of interest to classify those finite simple groups whose maximal chains are of length at most six. Thompson [8] has given the following

Definition. We say that a finite group $G$ is an $N$-group if the normalizer of any nontrivial solvable subgroup is itself solvable.

In the Main Theorem of [8], all simple $N$-groups are classified. These are the following groups: $\operatorname{PSL}(2, q), q$ a prime power greater than $3, \operatorname{PSL}(3,3), \mathrm{M}_{11}, \mathrm{~A}_{7}, \mathrm{Sz}\left(2^{2 n+1}\right)$ and $\operatorname{PSU}\left(3,3^{2}\right)$. Since these groups have been studied elsewhere in great detail, and have been variously characterized group theoretically, we will consider these groups as known. Then we have the

THEOREM. Let $G$ be a finite simple group all of whose sixth maximal subgroups are trivial. Then either $G$ is an $N$-group or $G \cong J$.

REMARK. It is easy to check that the only simple $N$-groups all of whose sixth maximal subgroups are trivial are the groups $\operatorname{PSL}(2, q)$ for certain prime powers $q$ or the group $\mathrm{A}_{7}$.

Proof of ThE Theorem. If $G$ is not an $N$-group, then $G$ contains a solvable subgroup $S \neq 1$ whose normalizer $N=N(S)$ is nonsolvable. All maximal chains of subgroups of $N$ have length at most 5. Thus all maximal chains of $N / S$ have length at most 4 . Since $N / S$ is nonsolvable, Theorems 1 and 2 of Janko [4] show that $N / S$ is simple and $N / S \cong \operatorname{PSL}(2, p)$, for some prime $p>3$. There is at least one chain of subgroups of length precisely 4 and so $S$ is cyclic of prime order. Also

Received by the editors July 27, 1967.

${ }^{1}$ Supported by NSF grant GP 6132. 
we note that $p$ is such that $p-1$ and $p+1$ are products of at most 3 primes and $p \equiv \pm 3(\bmod 8)$. Thus an $S_{2}$-subgroup of $N / S$ has order 4 .

Now since $S \leqq N(S), C(S) \leqq N(S)$ and $C(S) \geqq S$. Since $N / S$ is simple, either $C(S)=S$ or $C(S)=N(S)$. However, if $C(S)=S$, then $N / S$ is a subgroup of the automorphism group of $S$, which is cyclic of order $s-1$. This contradicts the simplicity of $N / S$. It follows that $s$ divides $p\left(p^{2}-1\right) / 2=|N / S|$, for otherwise, $S$ is an $S_{s}$-subgroup of $G$ and $G$ is nonsimple by a thoerem of Burnside [3, p. 204].

Let $R$ be an $S_{2}$-subgroup of $N,|R| \geqq 4$. We apply the following result of Schur, [9].

Let $N$ be a finite group and let $S$ be a subgroup of $N$ satisfying the following conditions:

(a) $1 \neq S \leqq Z(N)$,

(b) $N / S \cong \mathrm{PSL}(2, p), p>2, p$ a prime,

(c) $S \leqq[N, N]$.

Then $|S|=2$ and $N \cong \operatorname{SL}(2, p)$.

Thus we have that either $S \leqq[N, N]$ or $S \cap[N, N]=1$ since $S$ is of prime order. In the first case we have $|S|=2$ and $N \cong \operatorname{SL}(2, p)$. But then an $S_{2}$-subgroup $R$ of $N$ is a quaternion group of order 8 . Since the centre of $R$ is cyclic, $R$ is also an $S_{2}$-subgroup of $G$. But a group with a quaternion $S_{2}$-subgroup is not simple by the theorem of Brauer-Suzuki [1], a contradiction. Thus $S \cap[N, N]=1$, $S \times[N, N] \unlhd N$ and so $N=S \times[N, N] \cong S \times \operatorname{PSL}(2, p)$.

If $S$ is of odd order, $|R|=4$ and $R$ is not an $S_{2}$-subgroup of $G$. For all groups whose $S_{2}$-subgroups have order 4 are $N$-groups by the theorem of Gorenstein and Walter [2]. Consider $M=N(R)$. This group has order divisible by $2^{3} \cdot 3 \cdot s$ since $|N(R) \cap N(S)|=2^{2} \cdot 3 \cdot s$. Now every fifth maximal subgroup of $M$ is trivial and so every third maximal subgroup of $M / R$ is trivial. By a result of Huppert, [4, Theorem II], $M / R$ is solvable. It follows easily that $|M / R|$ $=2 \cdot 3 \cdot s, \quad|M|=2^{3} \cdot 3 \cdot s$. Now $|N(R) \cap N(S)|=2^{2} \cdot 3 \cdot s \quad$ and so $[N(R): N(R) \cap N(S)]=2$. Thus $N(R) \cap N(S) \unlhd N(R)$. Also $C(R)$ $\cap N(S)=S \times R$ and $C(R) \leqq N(R)$. Hence $C(R) \cap N(S) \leqq N(R) \cap N(S)$. Now $S=Z(N(R) \cap N(S))$ and so $S$ is a characteristic subgroup of $N(R) \cap N(S)$. Also $R$ is a normal $S_{2}$-subgroup of $N(R) \cap N(S)$ and so is characteristic in $N(R) \cap N(S)$. Thus $S R$ char $N(R) \cap N(S)$ $\unlhd N(R)$. If $s \neq 2, S$ char $S R$ and so $S \unlhd N(R), N(R) \leqq N(S)$. This contradicts the fact that an $S_{2}$-subgroup of $N(R)$ is of order 8 . Hence $|S|=2$ and an $S_{2}$-subgroup of $N$ is elementary abelian of order 8 .

If $R$ is an $S_{2}$-subgroup of $G$, we may apply the results of Janko [6] and Janko-Thompson [7], to get a contradication if $p \neq 5$ and $G \cong J$ 
if $p=5$. Thus we may assume that an $S_{2}$-subgroup $T \geqq R$ of $N(R)$ is of order at least 16 . Since $T \neq C(S), T$ is nonabelian.

Since $|N(R) \cap N(S)|=2^{3} \cdot 3,|N(R)|$ is divisible by $2^{4} \cdot 3$. Now, as before, $N(R) / R$ has the property that every second maximal subgroup is trivial. Hence $N(R) / R$ is solvable and it follows that $|N(R) / R|=2 \cdot 3,|T|=2^{4}$. There are just two possible groups $T$ which contain elementary subgroups of order 8 .

(a) $T \cong D_{8} \times C_{2}$ : a direct product of a cyclic group of order 2 and a dihedral group of order 8 .

(b) $T \cong\left\langle a, b, c: a^{4}=b^{2}=c^{2}=1,[a, c]=b,[a, b]=[b, c]=1\right\rangle$.

Let $X$ be an $S_{3}$-subgroup of $N(R)$. Then if $N(R)$ is 2-closed, $X$ normalizes $T$. There are just 4 elements of order 4 in the case (a) and 8 elements of order 4 in the case (b). Thus in either case, there exists an element $y \in T$ of order 4 which is fixed by $X$. But then $\left[y^{2}, X\right]=1$ and since $y^{2} \in R \leqq T, y^{2} \in C(X) \cap R$. Thus $y^{2} \in S$ and $y \in C(S)$. This is a contradiction because $C(S)$ has no elements of order 4 . Therefore $N(R) / R \cong S_{3}$.

Now $N(X) \cap N(R) \neq C(X) \cap N(R)$ because otherwise $N(R)$ has a normal 3-complement and is 2-closed. Hence if $H$ is an $S_{2}$-subgroup of $N(X) \cap N(R), H \geqq S,|H| \geqq 4$. Now $n=[N(R): N(X) \cap N(R)]$ $\equiv 1(\bmod 3)$ by Sylow's theorems and so $n=1$ or 4 . Of course, $X \oiint N(R)$ because $X \nsubseteq N(R) \cap N(S)$. Therefore $n=4$ and $|H|=4$. It follows that $H \leqq C\left(\overline{(S)}\right.$ and $C(S) \geqq R, H$. Thus an $S_{2}$-subgroup of $C(S)$ has order at least 16 , a contradiction. The theorem is proved.

\section{REFERENCES}

1. R. Brauer and M. Suzuki, On finite groups of even order whose 2-Sylow group is a quaternion group, Proc. Nat. Acad. Sci. U.S.A. 45 (1959), 1757-1759.

2. D. Gorenstein and J. H. Walter, The characterization of finite groups with dihedral 2-Sylow subgroups, J. Algebra 2 (1965), 85-151, 218-270, 354-393.

3. M. Hall, The theory of groups, Macmillan, New York, 1959.

4. Z. Janko, Finite groups with invariant fourth maximal subgroups, Math. Z. 82 (1963), 82-89.

5. - Finite groups with short chains of subgroups, Math. Z. 84 (1964), 428-437.

6. - A new finite simple group with abelian Sylow 2-subgroups and its characterization, J. Algebra 3 (1966), 147-186.

7. Z. Janko and J. G. Thompson, On a class of finite simple groups of Ree, J. Algebra 4 (1966), 274-292.

8. J. G. Thompson, Nonsolvable finite groups all of whose local subgroups are solvable, Bull. Amer. Math. Soc. 74 (1968), 383-437.

9. I. Schur, Untersuchungen ïber die Darstellung der endlichen Gruppen durch gebrochene lineare Substitutionen, J. für Math. 132 (1907) 85-137.

UNIVERSITY OF ILLINOIS 